\title{
Influence of Thickness and Magnetic Field on Magnetic Ordering Temperatures in Holmium Thin Films
}

\author{
J. DudÁS̆ $\check{S}^{a}, \mathrm{~S} . \mathrm{GABÁNI}^{b}, \mathrm{I}$. GoŠCIAŃSKA ${ }^{c}, \mathrm{~V} \cdot \mathrm{KAVEČANSKÝ}^{b}$,
}

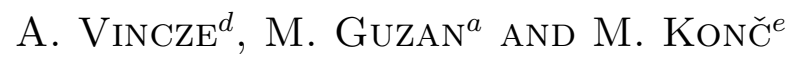

${ }^{a}$ Dep. of Theoretical Electrotechnics and Electrical Measurement Technical University, Park Komenského 3, 04389 Košice, Slovakia

${ }^{b}$ Institue of Experimental Physics, Slovak Academy of Sciences 04353 Košice, Slovakia

${ }^{c}$ Institute of Physics, A. Mickiewicz University Umultowska 85, 61-614 Poznań, Poland

${ }^{d}$ International Laser Centre, Ilkovičova 3, 81219 Bratislava, Slovakia ${ }^{e}$ Institute of Physics, P.J. Šafárik University Park Angelinum, 04154 Košice, Slovakia

Influence of thickness and magnetic field on magnetic ordering temperatures is reported. The X-ray diffraction of Ho films confirmed their preferential crystal orientation and revealed diffraction peaks originating from the hcp structure of Ho and those from inessential holmium dihydride content. Secondary ion mass spectroscopy showed a very homogeneous distribution of holmium in thin films. Electrical resistance measurements on Ho films in the thickness range from $98 \mathrm{~nm}$ to $215 \mathrm{~nm}$ showed a "knee-like" resistance anomaly near the $T_{\mathrm{N}}$. The $T_{\mathrm{N}}$ value of these films decreased with decreasing film thickness. Magnetic field applied parallel to the thin film plane caused an increasing suppression of the $T_{\mathrm{N}}$ value up to $5 \mathrm{~K}$ with increasing flux density value up to $5 \mathrm{~T}$.

PACS numbers: 73.61.At, 75.70.Ak, 68.55.Nq

\section{Introduction}

Thin rare-earth (RE) films attracted attention because of their magnetic properties (see e.g. [1]). Observations of new magnetic phenomena such as the giant magnetoresistance, attractive also for technical applications, revived the interest in thin magnetic films. Size effects may produce relevant modifications in the known magnetic phases of RE elements because of nonuniform magnetic structure 
induced by the lower coordination near the surfaces. Thickness effects on the magnetic ordering temperatures, magnetoelastic stresses and electric charge anomalies in Ho thin films were studied [2-5].

Holmium, like several other heavy lanthanide metals [6], orders magnetically by indirect exchange between the localized $4 f$ moments (Ruderman-KittelKasuya-Yosida (RKKY) interaction). Below $T_{\mathrm{N}}=130 \mathrm{~K}$ Ho orders antiferromagnetically in simple helical spin structure with magnetic moments in the basal plane. Below $T_{\mathrm{C}}=19 \mathrm{~K}$ the spins tilt out of the basal plane to form a conical structure.

The influence of film thickness and magnetic field on the magnetic ordering temperature $T_{\mathrm{N}}$ in Ho films is presented in this paper.

\section{Experimental}

Thin films of Ho with thickness of 98, 116, 147, 196, and $215 \mathrm{~nm}$ were evaporated onto the glass substrates in ultrahigh vacuum $\left(\approx 10^{-7} \mathrm{~Pa}\right)$. Electrical resistance of bulk and thin film samples was measured in helium cryostat by the conventional four-point dc arrangement using Keithley digital instruments. The temperature of the bulk and thin film samples was measured using calibrated Ge (from $4.2 \mathrm{~K}$ to $80 \mathrm{~K}$ ) and $\mathrm{Pt}$ (from $80 \mathrm{~K}$ up to $300 \mathrm{~K}$ ) thermometers. Film thickness was measured using the optical interference Tolansky method. The crystal structure of Ho films was determined using X-ray diffraction with the Bragg-Brentano focusing geometry. The purity and composition of the samples was studied using secondary ion mass spectroscopy (SIMS).

\section{Results and discussion}

The electrical resistance of the reference Ho bulk sample was measured prior to the Ho thin film study. The "knee-like" resistance anomaly was clearly seen near $\approx 130 \mathrm{~K}$. The numerical analysis of the resistance data yielded the $\mathrm{d} R^{2} / \mathrm{d} T^{2}$ vs. $T$ dependence with the deep minimum corresponding to the Néel temperature $T_{\mathrm{N}}=128.90 \mathrm{~K}$. Other minimum corresponding to the transition from spiral spin structure to cone one was found at $T_{\mathrm{C}}=19 \mathrm{~K}$.

The influence of thickness and temperature on the electrical resistance of Ho thin films was investigated in the temperature range from $4.2 \mathrm{~K}$ up to the room temperature in zero magnetic field and in the field with flux density from $1 \mathrm{~T}$ up to $5 \mathrm{~T}$. The result of this investigations is illustrated in Fig. 1 for film with thickness of $196 \mathrm{~nm}$. The $R$ vs. $T$ dependence of this Ho film exhibits a change of the resistance anomaly from a small "hump-backed" anomaly near $T_{\mathrm{N}}$ in zero magnetic field to a "knee-like" anomaly in magnetic field of $5 \mathrm{~T}$. It is illustrated in narrower temperature range near $T_{\mathrm{N}}$ in fields $0,1,2,3,4$, and $5 \mathrm{~T}$ in Fig. 2.

The $R$ vs. $T$ curves of other Ho films exhibit similar resistance anomalies in the low temperature region. The observed $R$ vs. $T$ dependence of Ho films under influence of magnetic field is the result of superposition of two temperature dependent conduction electron scattering mechanisms. According to Bloch-Grüneisen 

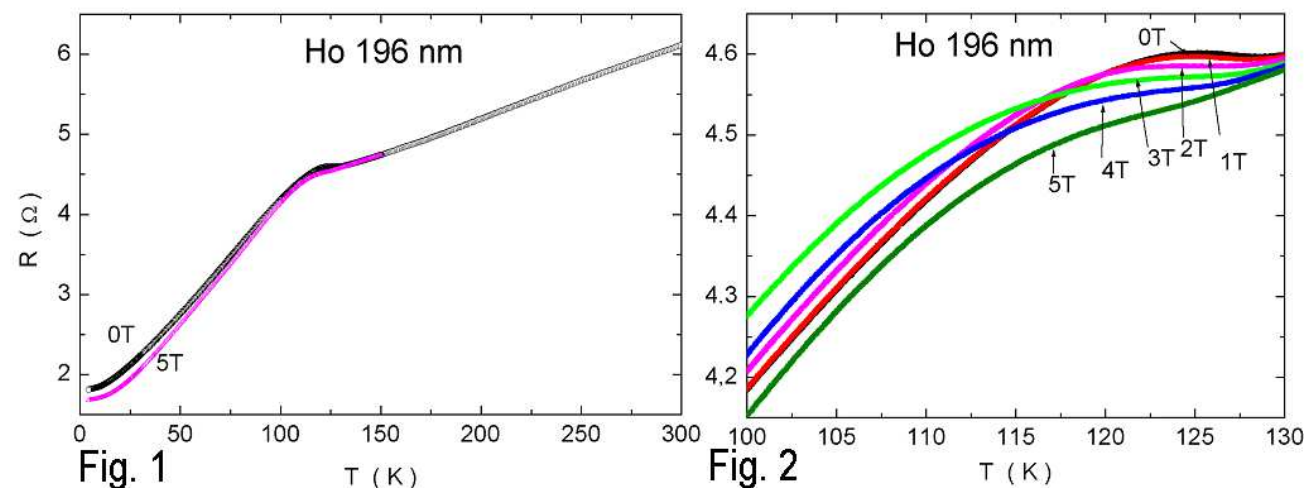

Fig. 1

$\mathrm{T}(\mathrm{K})$

Fig. 2

Fig. 1. The $R$ vs. $T$ dependence of the $196 \mathrm{~nm}$ thin Ho film in the temperature range from $4.2 \mathrm{~K}$ up to $300 \mathrm{~K}$ in zero and in magnetic field of $5 \mathrm{~T}$ below $150 \mathrm{~K}$.

Fig. 2. Influence of magnetic field on the $R$ vs. $T$ anomaly in $196 \mathrm{~nm}$ thin Ho film near $T_{\mathrm{N}}$ and in fields with increasing flux density value from $1 \mathrm{~T}$ up to $5 \mathrm{~T}$.
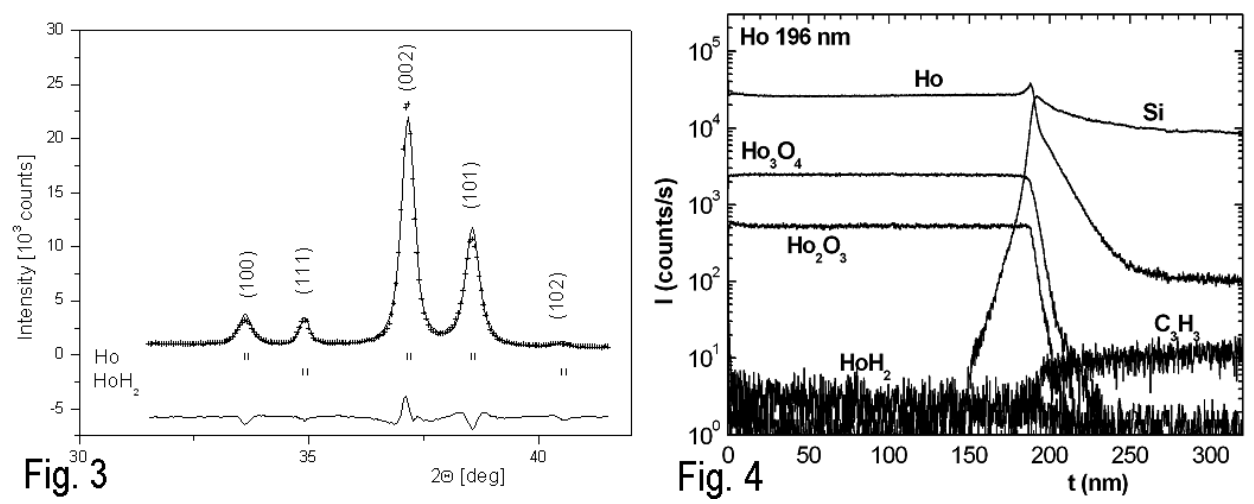

Fig. 3. The X-ray diffraction pattern for $196 \mathrm{~nm}$ thin Ho film.

Fig. 4. The SIMS depth profile of $196 \mathrm{~nm}$ thin Ho film with spectral lines of Ho, $\mathrm{Ho}_{3} \mathrm{O} 4, \mathrm{Ho}_{2} \mathrm{O}_{3}, \mathrm{HoH}_{2}, \mathrm{C}_{3} \mathrm{H}_{3}$, and $\mathrm{Si}$.

equation the electron-phonon interaction leads to resistance decrease with the change from $T^{1}$ dependence at higher temperatures to $T^{5}$ one at low temperatures. The spin scattering is influenced by the decrease in the spin turn angle below $T_{\mathrm{N}}$. The majority of the hcp crystallites of Ho is oriented with their basal plane parallel to the substrate (see Fig. 3). In lower fields oriented in the basal plane the helix is slightly distorted. Helifan phase appears, spins are more oriented in the direction of the applied field and spin scattering of the conduction electrons is lower. Upon further increase in the applied field the fan phase covers the whole film [1], thus the spin contribution to resistance decreases with increasing magnetic field. 
We have observed the decrease in the $T_{\mathrm{N}}$ value with decreasing film thickness, e.g. from $T_{\mathrm{N}}=126.90 \mathrm{~K}(196 \mathrm{~nm})$ to $T_{\mathrm{N}}=121.70 \mathrm{~K}(98 \mathrm{~nm})$. Reduced magnetic ordering temperatures have been observed in other RE metal thin films like Dy, Sm, Tm (see e.g. [7]) as well as in ferromagnetic films (see e.g. citations in [8]). This reduction is due to relative increase in internal stresses and impurities with decreasing film thickness and as the result of the intrinsic property of films.

Applied magnetic field caused a suppression of the $T_{\mathrm{N}}$ value - e.g. it decreased from $126.90 \mathrm{~K}(0 \mathrm{~T})$ to $122.50 \mathrm{~K}(5 \mathrm{~T})$ in $196 \mathrm{~nm}$ film and from $121.70 \mathrm{~K}$ $(0 \mathrm{~T})$ to $118.00 \mathrm{~K}(5 \mathrm{~T})$ in $98 \mathrm{~nm}$ film. We assume that the decrease in the $T_{\mathrm{N}}$ value in dc magnetic field is connected with nonuniform distortion of helix [1] which could lead to the decrease in the total magnetic exchange energy.

The crystal structure was investigated in all Ho films. Diffraction pattern of film with thickness $196 \mathrm{~nm}$ is shown in Fig. 3. The analysis revealed the (002) preffered orientation of the Ho films as well as the presence of the second but minority phase of holmium dihydride for all investigated samples. It is known that $\mathrm{RE}$ metal thin films readily react with hydrogen to form dihydrides with the fcc structure (see e.g. [7]) and/or the solid solution with the RE metals. Comparing the $R$ vs. $T$ curves of Ho films with those of bulk Ho we assume, the amount of HoH2 did not influence the $R$ vs. $T$ curve qualitatively.

The depth profiles SIMS spectra taken from the $196 \mathrm{~nm}$ Ho film are displayed in Fig. 4. These results show that the film has a homogeneous surface. The depth profile signals from $\mathrm{HoH}_{2}, \mathrm{Ho}_{2} \mathrm{O}_{3}, \mathrm{Ho}_{3} \mathrm{O}_{4}$ were present together with signals from other impurities. Nevertheless, the content of these contaminants was very low and they did not influence the transport properties of the films.

\section{Conclusions}

The following conclusions could be made from this study. (a) The X-ray diffraction studies of thin films revealed the (002) textured hcp Ho phase as well as the presence of the second but minority phase of fcc $\mathrm{HoH}_{2}$. (b) The SIMS depth profile showed the presence of $\mathrm{H}, \mathrm{HoH}_{2}, \mathrm{Ho}_{2} \mathrm{O}_{3}, \mathrm{Ho}_{3} \mathrm{O}_{4}$, organic components related to $\mathrm{C}_{x} \mathrm{H}_{y}$ ions, and other contaminants. However, their influence on the transport properties of thin films appeared to be negligible. (c) The $R$ vs. $T$ dependences of Ho films exhibit anomalies caused by magnetic structure similar to those observed in the Ho bulk sample. (d) The $T_{\mathrm{N}}$ values decreased with decreasing film thickness (e) Increasing magnetic field up to $5 \mathrm{~T}$ caused increasing suppression of the $T_{\mathrm{N}}$ value by about $5 \mathrm{~K}$.

\section{Acknowledgments}

The research was supported by the Slovak Scientific Grant Agency VEGA under projects 1/3139/06, 1/0130/03 ,2/4061/04,2/4050/04 and by the contract I/2/2003 of the Slovak Academy of Sciences for the Centres of Excellence. 


\section{References}

[1] V.D. Mello, C.V. Chianca, A.L. Dantas, A.S. Carriço, Phys. Rev. B 67, 012401 (2003).

[2] V. Leiner, D. Labergerie, R. Siebrecht, Ch. Sutter, H. Zabel, Physica B 283, 167 (2000).

[3] J.I. Arnaudas, M. Ciria, C. de la Fuente, L. Benito, A. del Moral, Low Temp. Phys. 27, 249 (2001).

[4] A.D.F. Herring, W.J. Nuttall, M.F. Thomas, J.P. Goff, A. Stunault, R.C.C. Ward, M.R. Wells, W.G. Stirling, J. Phys., Condens. Matter 17, 2543 (2005).

[5] J. Dudas, M. Guzan, S. Gabani, V. Kavečanský, K. Flachbart, M. Konč, I. Gościańska, Czech. J. Phys. 54, Suppl. D 253 (2004).

[6] W.C. Koehler, in: Magnetic Properties of Rare Earth Metals, Ed. R.J. Elliott, Plenum Press, London 1972, p. 81.

[7] J. Dudáš, Intricate Behaviour of Rare Earth Metal Thin Films at Low Temperatures, Mercury-Smékal, Košice 2001, p. 125.

[8] E. Weschke, H. Ott, E. Schierle, C. Schlüßler-Langeheine, D.V. Vyalikh, G. Kaindl, V. Leiner, M. Ay, H. Zabel, P.J. Jensen, Phys. Rev. Lett. 93, 157204 (2004). 